\title{
Nonrelativistic closed string theory
}

\author{
Jaume Gomis ${ }^{\text {a) }}$ and Hirosi Oogurib) \\ California Institute of Technology 452-48, Pasadena, California 91125 \\ and Caltech-USC Center for Theoretical Physics, Los Angeles, California
}

(Received 2 January 2001; accepted for publication 13 February 2001)

We construct a Galilean invariant nongravitational closed string theory whose excitations satisfy a nonrelativistic dispersion relation. This theory can be obtained by taking a consistent low energy limit of any of the conventional string theories, including the heterotic string. We give a finite first order worldsheet Hamiltonian for this theory and show that this string theory has a sensible perturbative expansion, interesting high energy behavior of scattering amplitudes and a Hagedorn transition of the thermal ensemble. The strong coupling duals of the Galilean superstring theories are considered and are shown to be described by an elevendimensional Galilean invariant theory of light membrane fluctuations. A new class of Galilean invariant nongravitational theories of light-brane excitations are obtained. We exhibit dual formulations of the strong coupling limits of these Galilean invariant theories and show that they exhibit many of the conventional dualities of M theory in a nonrelativistic setting. (C) 2001 American Institute of Physics.

[DOI: $10.1063 / 1.1372697$ ]

\section{INTRODUCTION}

One of the legacies of the second superstring revolution is the realization that the different superstring theories describe very special corners of the space of vacua of a single hypothetical structure dubbed M Theory. Another important lesson that has emerged is that there are regions of the space of vacua describable by a theory without gravity. Two beautiful examples of such theories are Matrix Theory ${ }^{1}$ and Maldacena's conjecture. ${ }^{2}$ The realization that there are consistent limits of M Theory without gravity has led to a geometrical understanding of some field theory dualities and to new, hitherto unknown, field theories in higher dimensions.

The nongravitational limits studied thus far involve considering certain low energy limits of M Theory in the presence of branes. Typically, these limits lead to a theory where the appropriate effective description is given in terms of the massless degrees of freedom propagating on the branes. Such low energy limits lead, for example, to gauge theories in various dimensions. In such examples, the massive open string states on the branes and the entire closed string spectrum decouple from the low energy physics and the truncation to the theory of the massless fluctuations is consistent. These low energy theories are described by field theories.

Recently, very interesting generalizations have been found in which closed strings decouple but the massive open string excitations on the branes need to be taken into account for physical processes. ${ }^{3,4}$ These theories appear in low energy limits of branes in near critical electric field backgrounds and are not conventional field theories due to the presence of a tower of massive excitations. Since massive states cannot be neglected, the field theory truncation is not unitary. ${ }^{5-7}$ These nongravitational theories describe all the fluctuations on the branes. For example, one can obtain a consistent open string theory without any closed string states. Such theories arise from studying D-branes in a background electric field (NCOS), ${ }^{3,4}$ M5-branes in a three-form background $(\mathrm{OM})^{8}$ and Neveu-Schwarz five-branes in various constant Ramond-Ramond $p$-form backgrounds $(\mathrm{OD}){ }^{8-10}$

\footnotetext{
${ }^{a)}$ Electronic mail: gomis@theory.caltech.edu

${ }^{b)}$ Electronic mail: ooguri@theory.caltech.edu
} 
In this paper we find that there are corners of the moduli space of vacua of M Theory without branes that are described by nongravitational theories whose excitations live in space-time. These massive excitations satisfy a nonrelativistic dispersion relation and the theory that describes their dynamics is unitary and has a sensible perturbative description (whenever one is available). Since background branes are not required to define these nonrelativistic theories, they can be obtained by taking certain low energy limits of all five superstring theories, including the heterotic string. We will call these theories nonrelativistic closed string theories (NRCS).

The simplest limit leading to NRCS is obtained by considering string theory in the presence of a near critical NS-NS two-form field without any D-brane (when the NS-NS two-form exceeds the critical value, the space-time energy of a closed string becomes unbounded below and can become indefinitely negative as we increase the winding number). In the context of $(1+1)$-dimensional NCOS, Klebanov and Maldacena ${ }^{11}$ observed that when the spatial direction is compactified on a circle, that there are finite energy winding closed string states that do not decouple from the open strings. An example of NRCS can be obtained by considering precisely the NCOS limit without any D-brane. Naively, one might think that, in the absence of D-branes, that a constant NS-NS two-form can be gauged away and that one ends up getting a conventional relativistic closed string theory. This is obviously true in noncompact space. However, in the presence of a circle, the background NS-NS field modifies the spectrum, which remains relativistic. Once the NCOS limit is taken, there is a truncation of the low energy spectrum and one obtains a new theory with a Galilean invariant Hamiltonian. Perhaps surprisingly, the closed string theory in the NCOS limit without any D-brane has a well-behaved perturbative expansion, described by the Lagrangian in Sec. III. It is also interesting to study the worldsheet theory we propose when the worldsheet has a boundary. Then, our formalism reproduces the relativistic open string spectrum of NCOS and its interactions.

In Sec. III, we give a worldsheet Lagrangian for NRCS, which has Galilean invariance and from which we derive the nonrelativistic spectrum of closed strings and their interactions. The Lagrangian we propose can be derived from the conventional Polyakov path integral quantization of the relativistic string by rewriting it in variables that are conducive to taking the low energy limit that defines NRCS (see Sec. III for details of the limit). We explicitly solve the Virasoro constraints, thus yielding the spectrum, show that the theory is unitary and that it has a sensible perturbative expansion. The string spectrum, being nonrelativistic, does not contain a massless graviton and it is thus nongravitational in nature. However, there is an instantaneous Newtonian potential between the massive strings. This string theory exhibits interesting properties such as an unusual high energy behavior of scattering amplitudes and a Hagedorn transition of the thermal ensemble.

NRCS depends on two parameters, the effective string scale $\alpha_{\text {eff }}^{\prime}$ and the effective string coupling constant $g$. One may ask what is the strong coupling dual of these theories. For the superstrings, this can be reliably answered. We find that the strong coupling limits of supersymmetric NRCS are given by a Galilean invariant eleven-dimensional theory of light membranes which we call GM (Galilean membrane theory). This eleven-dimensional theory has a unique dimensionful parameter $l_{\text {eff }}$ which is the effective Planck length. The relation between the NRCS superstrings and GM is reminiscent to the relation between the conventional superstrings and $\mathrm{M}$ theory. For example, Type IIA NRCS with coupling $g$ and string scale $\alpha_{\text {eff }}^{\prime}$ is equivalent to GM on a circle of radius $R$ such that $R=g \sqrt{\alpha_{\text {eff }}^{\prime}}$ and $l_{\text {eff }}=g \sqrt{1 / 3} \sqrt{\alpha_{\text {eff }}^{\prime}}$. The conventional dualities and relations with M theory still hold, such that, for example. Type IIB NRCS has an $S L(2, Z)$ symmetry. We discuss these relations in Sec. VII. It is interesting that duality symmetries in string theories do not rely on relativistic invariance nor the presence of gravity.

There are many interesting generalizations that can be made that lead to nonrelativistic, nongravitational theories. The construction of such theories is quite general. The basic idea is to study the low energy limit of M Theory vacua in the presence of any of the many possible gauge fields available. Then, one can take a low energy, near critical limit such that all states of $\mathrm{M}$ Theory become infinitely massive, and thus decouple, except for those states that couple to the constant near critical background gauge field. Tuning to the critical value, defined such that that 
energy coming from the background field precisely cancels the rest energy of the states in question, ensures that even though we are taking a low energy limit, that there are states that survive and satisfy a nonrelativistic dispersion relation. For example, if we tune the background NS-NS B field to its critical value, then one obtains finite energy nonrelativistic fluctuations of strings winding around the circle. Clearly, such NRCS can be defined in Type II, Type I and heterotic theories. Moreover, if one considers, for example, a near critical $\mathrm{R}-\mathrm{R}$ gauge field $C_{p+1}$ and takes a low energy limit, then there are light Dp-branes (in order for the constant background $C_{p+1}$ field to affect the energy of a Dp-brane, the brane has to be wrapped on a $p$-cycle, otherwise the gauge field can be gauged away without changing the energetics) which are nonrelativistic that decouple from all the rest of the modes and lead to decoupled Galilean invariant theories which we will call GDp (Galilean Dp-brane theories). The myriad of gauge fields that exist in M Theory vacua can be used to define new nongravitational Galilean invariant theories. We study such theories in Sec. VII. The dualities of the underlying relativistic M Theory, lead to interesting webs of dualities for these nonrelativistic theories. These nonrelativistic theories may be a promising ground in which to address some of the important questions of M Theory without the complication of gravity.

The rest of the paper is organized as follows.

In Sec. II a very general low energy limit is presented which yields a finite nonrelativistic dispersion relation from the spectrum of a charged relativistic brane. The limit, when applied to the fundamental closed string, yields the spectrum of NRCS. Generalizations to other relativistic objects in M Theory are briefly described.

In Sec. III we find the worldsheet theory of NRCS. We quantize the Galilean invariant, first order Hamiltonian and find under what conditions there is a physical closed string spectrum. We then reproduce the NRCS spectrum in Sec. II within our Hamiltonian formalism. We compute the BRST cohomology of the string and show that there are no ghosts in the spectrum. The possibility of adding a boundary to the worldsheet is considered. The formalism of Sec. III results in the spectrum and worldsheet correlation functions of NCOS. Using this formalism, it is straightforward to prove the decoupling of the massless open string states on worldsheets with any number of handles and holes when the longitudinal direction is noncompact. This extends the result of Ref. 11 to all orders in the perturbative expansion.

Section IV is devoted to performing tree level computations in NRCS. We show that scattering amplitudes have the correct pole structure required by unitarity and have a peculiar behavior of high energy fixed angle scattering amplitudes in NRCS. Despite the absence of gravity in this theory, we exhibit a Newtonian potential among the nonrelativistic strings.

In Sec. V we compute loop amplitudes and show that NRCS is a sensible theory in perturbation theory. We evaluate the Helmholtz free energy at one loop and reproduce from it the spectrum of NRCS found in Sec. II. We find that NRCS behaves similarly to the long string near the boundary of $A d S_{3} .{ }^{12}$ We exhibit the existence of a Hagedorn temperature in NRCS and sketch higher loop computations. We also study in some detail $N$-point loop amplitudes and show that the amplitudes are finite.

In Sec. VI we elucidate the relation between NRCS and the discrete light-cone quantization (DLCQ) of closed string theory. NRCS is related by T-duality to the discrete light-cone quantization (DLCQ) of closed string theory. Therefore, the formalism developed in this paper provides a useful description of DLCQ string theories as well.

In Sec. VII we study Galilean invariant theories of light branes and some of their dualities. In eleven dimensions we study the Galilean invariant theory of membrane fluctuations (GM) and five-brane fluctuations (GF). In ten dimensions we discuss the theory of nonrelativistic light Dp-branes (GDp) and light Neveu-Schwarz five-branes (GNS). These theories lie in the same moduli space and exhibit the same dualities that the underlying relativistic $\mathrm{M}$ theory possesses. In particular we show that the strong coupling limits of some NRCS have an eleven-dimensional description in terms of light-brane excitations. 


\section{NONRELATIVISTIC LIMIT}

In this section we show that by taking a low energy limit of the theory of a relativistic $p$-brane and by tuning the $p+1$ gauge field that couples to it, that one can obtain an exact nonrelativistic dispersion relation. The idea is to study the low energy spectrum in a scaling limit in which the background gauge field cancels the rest energy of the brane and such that the nonrelativistic approximation becomes exact. In this limit, all the states of the theory decouple, except the light $p$-brane excitations. We present the truncation to a nonrelativistic theory in a very simple toy model which captures the essence of the limit which defines NRCS and the other generalizations we describe in this paper.

For simplicity, consider a relativistic charged point particle of mass $m$ and charge $e$ coupled to a gauge field $A_{\mu}$ propagating in a geometry with metric components $g_{00}=-1$ and $g_{i j}$ arbitrary with $i, j \neq 0$. The Lagrangian which describes its motion is given by

$$
L=-m \sqrt{-\dot{x}^{2}}+e A_{\mu} \dot{x}^{\mu}
$$

Worldline reparametrization invariance implies Einstein's dispersion relation,

$$
p_{0}=\sqrt{m^{2}+g^{i j}\left(p_{i}-e A_{i}\right)\left(p_{j}-e A_{j}\right)}+e A_{0} .
$$

Consider the following low energy limit:

$$
g_{i j}=\frac{m_{\mathrm{eff}}}{m} \delta_{i j}, \quad e A_{0}=-m+e \hat{A}_{0},
$$

as $m \rightarrow \infty$. In this limit, Einstein's relation (2.2) reduces to the following nonrelativistic dispersion law:

$$
p_{0}=\frac{1}{2 m_{\mathrm{eff}}}\left(p_{i}-e A_{i}\right)^{2}+e \hat{A}_{0} .
$$

Although a constant gauge field can be locally gauged away and does not affect the equations of motion, it changes the energy spectrum in the sector of the theory carrying electric charge. In fact, the shift in the energy due to the gauge field precisely cancels the rest mass of the particle and ensures that the energy remains finite in the limit (2.3). Turning on a background field and tuning it to the critical value is an efficient way of rearranging the spectrum of the theory such that only states charged under the gauge field have finite energy, the neutral states acquire infinite proper energy.

The charged point particle model can also be used to show that the there are finite energy, nonrelativistic winding closed string states in the NCOS limit whenever the near critical NS-NS $B_{01}$-field is along a compact spatial direction. The mass of a closed string winding $w$-times around a circle of radius $R$ is

$$
m^{2}=\left(\frac{w R}{\alpha^{\prime}}\right)^{2}+\frac{2(N+\widetilde{N})}{\alpha^{\prime}},
$$

where $N$ and $\bar{N}$ are the amounts of stringy excitations in the left and the right mover oscillators of the string. Moreover, the winding string states are charged under the $U(1)$ gauge field obtained by reducing the NS-NS $B$-field along the circle. The charge is given by

$$
e A_{0}=-2 \pi R w B_{01} \text {. }
$$

We now take the NCOS limit ${ }^{3,4}$ in the point particle analogy (2.4), 


$$
g_{i j}=\frac{\alpha^{\prime}}{\alpha_{\mathrm{eff}}^{\prime}} \delta_{i j}, \quad e A_{0}=-\frac{w R}{\alpha^{\prime}}\left(1-\frac{\alpha^{\prime}}{2 \alpha_{\mathrm{eff}}^{\prime}}\right)
$$

Taking $\alpha^{\prime} \rightarrow 0$ results in the following nonrelativistic spectrum:

$$
p_{0}=\frac{w R}{2 \alpha_{\mathrm{eff}}^{\prime}}+\frac{\alpha_{\mathrm{eff}}^{\prime} k^{2}}{2 w R}+\frac{N+\tilde{N}}{w R} .
$$

Thus the NCOS limit can be thought of an example of the nonrelativistic limit (2.3). Note that demanding positive energy states selects strings winding only in a particular direction. Indeed, the closed string spectrum (2.8) coincides with the one found by Maldacena and Klebanov in Ref. 11. In the next section we give a Galilean invariant, finite first order Hamiltonian that describes these closed strings and verify that there is a consistent perturbative expansion.

The nonrelativistic limit we found in (2.3) can also be generalized to any state of M Theory which is charged under a gauge field. If one considers winding fundamental closed strings in the near critical NS-NS $B_{0 i}$ background of any of the known string theories, one obtains NRCS theories. But we could have considered any of the branes of M Theory. One could, for example, consider a wrapped membrane of $\mathrm{M}$ Theory on any two-cycle (say on a two-torus or a two-cycle of a Calabi-Yau) in a critical three-form background. Then, the membrane is charged under a gauge field $A_{0}$ obtained by reducing the three-form on the two-cycle. In the limit (2.3) one obtains a nonrelativistic theory without gravity. Likewise, for any other brane. Just like in NCOS, positivity of the energy selects only those states which are wrapped in a particular direction, states of opposite orientation are unphysical. In Sec. VII we will find low energy limits leading to Galilean theories of branes and study their strong coupling duals.

\section{LAGRANGIAN AND QUANTIZATION}

In this section we construct the worldsheet theory of NRCS and analyze its spectrum and interactions. We consider a certain low energy limit of string theory in a near critical NS-NS $B$-field. The bosonic worldsheet action which describes this background is given by (here the worldsheet and target space metric are taken to be of Lorentzian signature)

$$
S_{0}=-\frac{1}{4 \pi \alpha^{\prime}} \int d^{2} \sigma\left(g_{M N} \partial_{a} X^{M} \partial^{a} X^{N}-2 \pi \alpha^{\prime} B_{M N} \epsilon^{a b} \partial_{a} X^{M} \partial_{b} X^{N}\right)
$$

where $M, N=0, \ldots, 9$ and $a, b=0,1$. NRCS is obtained by choosing the $B$-field with a time-like and a space-like component. Without loss of generality we consider a $B_{01} \equiv B$ background. NRCS is obtained by taking the following zero slope, near critical field limit (this is precisely the NCOS limit of Refs. 3, 4 but without any D-brane),

$$
2 \pi \alpha^{\prime} B_{01}=1-\frac{\alpha^{\prime}}{2 \alpha_{\mathrm{eff}}^{\prime}}, \quad g_{\mu \nu}=\eta_{\mu \nu}, \quad g_{i j} \frac{\alpha^{\prime}}{\alpha_{\mathrm{eff}}^{\prime}} \delta_{i j}, \quad g_{s}=g \sqrt{\frac{\alpha_{\mathrm{eff}}^{\prime}}{\alpha^{\prime}}},
$$

as $\alpha^{\prime} \rightarrow 0$ where $\mu, \nu=0,1$ and $i, j=2, \ldots 9$, and $\alpha_{\text {eff }}^{\prime}$ is the finite effective string scale of NRCS and $g$ its effective coupling constant.

Using

$$
\gamma=X^{0}+X^{1}, \quad \bar{\gamma}=-X^{0}+X^{1},
$$

for the target space coordinates,

$$
z=e^{i\left(\sigma^{0}+\sigma^{1}\right)}, \quad \bar{z}=e^{i\left(\sigma^{0}-\sigma^{1}\right)},
$$


for the worldsheet coordinates, and the background given in (3.2), the action (3.1) can be written for finite $\alpha^{\prime}$ as

$$
S_{0}=-\frac{1}{4 \pi \alpha^{\prime}} \int d^{2} z\left(\partial \gamma \bar{\partial} \bar{\gamma}+\partial \bar{\gamma} \bar{\partial} \gamma-2 \pi \alpha^{\prime} B(\partial \gamma \bar{\partial} \bar{\gamma}-\partial \bar{\gamma} \bar{\partial} \gamma)+2 g_{i j} \partial X^{i} \bar{\partial} X^{j}\right)
$$

We now perform a Euclidean rotation in both the worldsheet and target space such that the Euclidean action is

$$
S_{0}=\frac{1}{4 \pi \alpha^{\prime}} \int d^{2} z\left(\partial \lambda \bar{\partial} \bar{\gamma}+\partial \bar{\gamma} \bar{\partial} \gamma-2 \pi \alpha^{\prime} B(\partial \gamma \bar{\partial} \bar{\gamma}-\partial \bar{\gamma} \bar{\partial} \gamma)+2 g_{i j} \partial X^{i} \bar{\partial} X^{j}\right) .
$$

Note that, unlike the case of a $B$-field with only space-like components, there is no factor of $i$ in the term in the Euclidean action depending on $B$.

In order to obtain a finite worldsheet description in the NRCS limit, it is convenient to introduce Lagrange multipliers $\beta$ and $\bar{\beta}$. In these variables the worldsheet theory (3.6) is given by

$$
S_{1}=\int \frac{d^{2} z}{2 \pi}\left(\beta \bar{\partial} \gamma+\bar{\beta} \partial \bar{\gamma}-\frac{2 \alpha^{\prime}}{1+2 \pi \alpha^{\prime} B} \beta \bar{\beta}+\frac{1+2 \pi \alpha^{\prime} B}{2 \alpha^{\prime}} \partial \gamma \bar{\partial} \bar{\gamma}+\frac{1}{\alpha^{\prime}} g_{i j} \partial X^{i} \bar{\partial} X^{j}\right),
$$

where (3.6) is reproduced by integrating out $\beta$ and $\bar{\beta}$. Therefore, in the strict decoupling limit (3.2), one has the following Lagrangian description of NRCS:

$$
S_{1}=\int \frac{d^{2} z}{2 \pi}\left(\beta \bar{\partial} \gamma+\bar{\beta} \partial \bar{\gamma}+\frac{1}{4 \alpha_{\mathrm{eff}}^{\prime}} \partial \gamma \bar{\partial} \bar{\gamma}+\frac{1}{\alpha_{\mathrm{eff}}^{\prime}} \partial X^{i} \bar{\partial} X_{i}\right) .
$$

We note that the worldsheet theory of NRCS is defined in terms of more variables than a conventional critical string theory since we have extra $\beta$ and $\bar{\beta}$ variables. However, the CFT defined by (3.8) has the correct Virasoro central charge to define a consistent string action (see the next subsection for more details). It is interesting to note that the Lagrangian (3.8) is invariant under the Galilean group in the transverse coordinates. This is consistent with the nonrelativistic spectrum that we will find for NRCS. It is crucial, for this symmetry to be realized, that the description has the extra variables $\beta$ and $\bar{\beta}$.

We will concentrate on the conformal field theory of $\beta, \bar{\beta}$ and $\gamma, \bar{\gamma}$ since the transverse coordinates lead to familiar contributions. The equations of motion force $\beta$ and $\gamma$ to be holomorphic and $\bar{\beta}$ and $\bar{\gamma}$ to be antiholomorphic. The Lagrange multiplier $\beta$ forces $\gamma$ to be a holomorphic map from the worldsheet to the $(1+1)$-dimensional part of the target space parameterized by coordinates $X^{0}$ and $X^{1}$. Therefore, it describes a worldsheet instanton and the third term in the Lagrangian (3.8) $\left(1 / 8 \pi \alpha_{\text {eff }}^{\prime}\right) \partial \gamma \partial \bar{\gamma}$ is the instanton action. We will show in the rest of this section that this Lagrangian reproduces the spectrum in (2.8). Moreover, we will see that if the string worldsheet has a boundary that (3.8) reproduces the open string spectrum of NCOS and string interactions. In our formalism, one can show that the decoupling of the massless open string modes exhibited by Ref. 11 at the disk level can be extended to all orders in perturbation theory. Thus, (3.8) can be used to perform manifestly finite worldsheet computations for NCOS theories. In the later sections, we will use this Lagrangian to describe amplitudes involving closed strings and higher loops.

\section{A. Closed string spectrum}

Here we consider a worldsheet without boundaries. The equations of motion of (3.8) imply that $\beta(z)$ and $\gamma(z)$ are holomorphic and that $\bar{\beta}(\bar{z})$ and $\bar{\gamma}(\bar{z})$ are antiholomorphic. Their OPE's are given by 


$$
\begin{gathered}
\beta(z) \gamma(w) \sim \frac{-1}{z-w}, \quad \bar{\beta}(\bar{z}) \bar{\gamma}(\bar{w}) \sim \frac{-1}{\bar{z}-\bar{w}}, \\
\gamma(z) \beta(w) \sim \frac{1}{z-w}, \quad \bar{\gamma}(\bar{z}) \bar{\beta}(\bar{w}) \sim \frac{1}{\bar{z}-\bar{w}}, \\
\gamma(z) \bar{\gamma}(\bar{w}) \sim \text { regular, } \quad \beta(z) \bar{\beta}(w) \sim \frac{-\pi}{2 \alpha_{\mathrm{eff}}^{\prime}} \delta^{(2)}(z-w) .
\end{gathered}
$$

The variables $\beta$ and $\gamma$ behave analogously to the bosonic ghost system, except for the contact term in $\beta(z) \bar{\beta}(\bar{w})$. The conformal dimension of $\gamma$ is $(0,0)$ while the conformal dimension of $\beta$ is $(1$, $0)$. Moreover, their contribution to the Virasoro central charge takes the required value $c=2$. Note that although it seems that we have added more degrees of freedom to the description, the worldsheet degrees of freedom are identical to those of two worldsheet scalars. A similar story holds for the $\bar{\beta}$ and $\bar{\gamma}$ system.

We will first consider the case when the $x^{1}$ coordinate is noncompact. Then we can expand the operators as

$$
\gamma(z)=\sum_{n=-\infty}^{\infty} \gamma_{n} z^{-n}, \quad \beta(z)=\sum_{n=-\infty}^{\infty} \beta_{n} z^{-n-1}
$$

Since $\gamma$ is holomorphic and $x^{1}$ is noncompact the standard space-time momentum term in $\gamma$ is not allowed. Otherwise, $\gamma$ would be multi-valued function of $\sigma$, which is only possible if the string is winding. The oscillator modes satisfy the following commutation relation:

$$
\left[\gamma_{n}, \beta_{m}\right]=\delta_{n+m, 0}
$$

We now study the Virasoro constraints for NRCS. The energy momentum tensor is given by

$$
T(z)=-\beta \partial \gamma
$$

and the Virasoro generators are

$$
L_{n}=\sum_{m} m \beta_{n-m} \gamma_{m}
$$

In particular, $L_{0}$ is the excitation level of the $(\beta, \gamma)$ system, whose spectrum is positive definite. Therefore the Virasoro constraint $L_{0}+\widetilde{L}_{0}=1$ has no solutions (except for the tachyon, which is projected out in supersymmetric theories), where $\widetilde{L}_{0}$ is the Virasoro generator for the rest of the system, which we assume to be positive definite. Thus, the closed string has no physical states. This can be easily understood. If all coordinates are noncompact the background NS-NS $B$-field can be gauged away without changing the Hamiltonian of the theory and the closed string spectrum is the usual one. Therefore, in the NRCS limit (3.2), all the closed strings acquire infinite proper energy and thus are unphysical.

When the $x^{1}$ direction is compactified on a circle of radius $R$, there is a nonzero winding sector in $\gamma(z)$. The mode expansion now reads as

$$
\gamma(z)=i w R \log z+\sum_{n=-\infty}^{\infty} \gamma_{n} z^{-n}
$$

and the Virasoro generator is then given by 


$$
L_{n}=-i \beta_{n} w R+\sum_{m} m \beta_{n-m} \gamma_{m}
$$

Now the Virasoro constraint $L_{0}+\widetilde{L}_{0}=1$ has a solution. As we will show later, all physical states are in the vacuum of the $(\beta, \gamma)$-system. Thus the solution to the Virasoro constraint is of the form

$$
i \beta_{0}=\frac{N}{w R}+\frac{\alpha_{\mathrm{eff}}^{\prime} k^{2}}{4 w R}
$$

where $N$ is the conformal weight of the rest of the system ( $N$ includes -1 for the bosonic string and $-1 / 2$ for the NS sector of superstring) and $k$ is the transverse momentum of the string. According to the Lagrangian (3.8), the canonical momentum $P$ conjugate to $\gamma$ is not equal to $\beta$, but in the winding sector it is shifted by an amount proportional to $\bar{\partial} \bar{\gamma}$ as in

$$
\frac{1}{2}\left(P_{0}+P_{1}\right)=\frac{\beta}{2 \pi}-\frac{1}{8 \pi \alpha_{\text {eff }}^{\prime}} \bar{\partial} \bar{\gamma}, \quad \frac{1}{2}\left(P_{0}-P_{1}\right)=\frac{\bar{\beta}}{2 \pi}-\frac{1}{8 \pi \alpha_{\text {eff }}^{\prime}} \partial \gamma
$$

Taking the zero mode parts of these equations, one finds that the total energy $p_{0}$ and momentum $p_{1}$ of the closed string are given by

$$
\frac{1}{2}\left(p_{0}+p_{1}\right)=i \beta_{0}+\frac{1}{4 \alpha_{\mathrm{eff}}^{\prime}} w R, \quad \frac{1}{2}\left(p_{0}-p_{1}\right)=i \bar{\beta}_{0}+\frac{1}{4 \alpha_{\mathrm{eff}}^{\prime}} w R .
$$

Since $x^{1}$ is periodic, its conjugate momentum is quantized as

$$
p_{1}=\frac{n}{R}
$$

Thus we find

$$
p_{0}=\frac{w R}{2 \alpha_{\mathrm{eff}}^{\prime}}+\frac{\alpha_{\mathrm{eff}}^{\prime} k^{2}}{2 w R}+\frac{N+\bar{N}}{w R}
$$

with the level matching condition $N-\bar{N}=w n$. In this way, we have recovered the nonrelativistic spectrum in (2.8).

It is straightforward to prove the no-ghost theorem in this case. We introduce the $(b, c)$ ghost and write the BRST operator $Q_{\mathrm{BRST}}$ as

$$
Q_{\mathrm{BRST}}=Q_{-1}+Q_{0},
$$

where

$$
Q_{-1}=w R \sum_{n} \beta_{-n} c_{n}
$$

and $Q_{0}$ is defined as the remainder. They obey

$$
Q_{-1}^{2}=Q_{0}^{2}=\left\{Q_{-1}, Q_{0}\right\}=0 \text {. }
$$

Following the use of the BRST operator in Ref. 13 (see also Sec. 4.4 of Ref. 14), one can show that the cohomology of $Q_{\mathrm{BRST}}$ is isomorphic to that of $Q_{-1}$. The cohomology of the quadratic 
operator $Q_{-1}$ is easy to evaluate ${ }^{15}$ and it is spanned by the vacuum state $|0\rangle_{\beta \gamma} \otimes|0\rangle_{b c}$ of $(\beta, \gamma)$ and $(b, c)$, times any states in the rest of the system. Assuming that the rest of the system is unitary, this proves the no-ghost theorem of NRCS.

\section{B. Open string spectrum}

It is possible to consider D-branes in NRCS, and what one gets is of course NCOS. Here we show that the open string spectrum of NCOS is reproduced in this way. Suppose the worldsheet is the upper half plane with the boundary located at $\sigma^{1}=0$. In the bulk, $\gamma$ and $\beta$ are still holomorphic. The equation of motion at the boundary gives

$$
\delta \gamma\left(\beta+\frac{1}{4 \alpha_{\mathrm{eff}}^{\prime}} \bar{\partial} \bar{\gamma}\right)=0, \quad \delta \bar{\gamma}\left(\bar{\beta}+\frac{1}{4 \alpha_{\mathrm{eff}}^{\prime}} \partial \gamma\right)=0 .
$$

For a Dp-brane with $p \geqslant 1$, the boundary values of $(\gamma, \bar{\gamma})$ are not fixed. Therefore

$$
\beta=-\frac{1}{4 \alpha_{\mathrm{eff}}^{\prime}} \bar{\partial} \bar{\gamma}, \quad \bar{\beta}=-\frac{1}{4 \alpha_{\mathrm{eff}}^{\prime}} \partial \gamma
$$

at the boundary. This suggests that we analytically continue $(\gamma, \beta)$ to $\sigma^{1}<0$ and use (3.25) to identify $(\gamma, \beta)$ on $\sigma^{1} \geqslant 0$ to $(\bar{\gamma}, \bar{\beta})$ in $\sigma^{1} \leqslant 0$. Since

$$
\beta(z) \gamma(w) \sim-\frac{1}{z-w}, \quad \bar{\beta}(\bar{z}) \bar{\gamma}(\bar{w}) \sim-\frac{1}{\bar{z}-\bar{w}},
$$

we find

$$
\gamma(z) \bar{\gamma}(\bar{w}) \sim 4 \alpha_{\text {eff }}^{\prime} \log (z-\bar{w}) .
$$

For points on the real axis this reproduces the correct propagator for open strings in NCOS.

To compute the open string spectrum, we consider a worldsheet that is a strip $-\pi \leqslant \sigma^{1}$ $\leqslant \pi$, we identify $(\gamma, \beta)$ on $0 \leqslant \sigma^{1} \leqslant \pi$ to $(\bar{\gamma}, \bar{\beta})$ on $-\pi \leqslant \sigma^{1} \leqslant 0$ according to (3.25). Thus we have the expansion of these fields as

$$
\begin{aligned}
& \beta=\frac{1}{2 \sqrt{\alpha_{\mathrm{eff}}^{\prime}}} \sum_{n} \bar{\alpha}_{n} \bar{z}^{-n-1}, \quad \gamma=x+4 i p \alpha_{\mathrm{eff}}^{\prime} \log z+2 \sqrt{\alpha_{\mathrm{eff}}^{\prime}} \sum_{n \neq 0} \frac{\alpha_{n}}{n} z^{-n}, \\
& \bar{\beta}=\frac{1}{2 \sqrt{\alpha_{\mathrm{eff}}^{\prime}}} \sum_{n} \alpha_{n} z^{-n-1}, \quad \bar{\gamma}=\bar{x}+4 i \bar{p} \alpha_{\mathrm{eff}}^{\prime} \log \bar{z}+2 \sqrt{\alpha_{\mathrm{eff}}^{\prime}} \sum_{n \neq 0} \frac{\bar{\alpha}_{n}}{n} \bar{z}^{-n},
\end{aligned}
$$

and nonzero commutators are

$$
\left[\alpha_{n}, \bar{\alpha}_{m}\right]=n \delta_{n+m, 0}, \quad[x, \bar{p}]=[\bar{x}, p]=i .
$$

Note that, unlike the case of closed string, the space-time momentum term $4 i p \alpha_{\mathrm{eff}}^{\prime} \log z$ is allowed in $\gamma$ even when $x^{1}$ is noncompact. This is because we can choose the branch cut of $\log z$ to be away from the worldsheet. The Virasoro generators are then

$$
L_{n}=\sum_{m} \bar{\alpha}_{n-m} \alpha_{m}
$$

Thus we reproduce the standard open string spectrum. 


\section{Free the $U(1)$ to all order}

In Ref. 11, it was shown that any tree level amplitude containing a massless open string state in $\mathrm{NCOS}_{2}$ vanishes when the longitudinal direction is noncompact. This is what is expected from the point of view of the S-dual theory, where the massless open strings correspond to the free $U(1)$ gauge fields and their superpartners in the $(1+1)$-dimensional $U(N)$ gauge theory. Using the formalism developed here, it is straightforward to generalize this result to all orders in perturbation theory. The open string amplitude on a worldsheet with $h$ holes and $g$ handles is computed by considering a closed Riemann surface of genus $2 g$ with a complex conjugate involution such that the fixed point set of the involution gives the boundaries of the open string worldsheet. Since there is no vertex operator inserted away from the boundary (when the longitudinal direction is noncompact there is no closed string physical state), $\gamma(z)$ is holomorphic everywhere except at the boundaries. Moreover, the vertex operator for a massless open string state is also holomorphic (vertex operators for massive states are not holomorphic; they also depend on $\bar{\gamma}$ ). Since the sum of the boundaries of the $h$ holes obtained as the fixed point set of the involution is homologically trivial on the genus $2 g$ surface, the contour integral of the vertex operator can be deformed away through the middle of the Riemann surface. This proves the decoupling of the massless open string states to all order in the perturbation theory.

\section{TREE AMPLITUDES}

In this section we will compute the scattering amplitude of four physical closed string states and show that it factorizes properly into nonrelativistic closed string poles. Moreover, we will see that the truncated closed string scattering amplitudes have a different high energy behavior than in conventional string theory.

For simplicity, we will compute the 4-tachyon amplitude. Since all the physical states are in the vacuum of the $(\beta, \gamma)$-system as we saw in the last section, the essential novelty of NRCS is captured by the tachyon amplitude. The vertex operator for a closed string tachyon is given by (we will not include the cocycles which only change relative signs between amplitudes; the factor $\sqrt{w}$ is included for later convenience)

$$
V(v, \bar{v}, k ; z, \bar{z})=g \sqrt{w}: e^{i \bar{v} \gamma(z)+i w R \int^{z} \beta+i v \bar{\gamma}(\bar{z})+i w R \int^{\bar{z}} \beta+i k \cdot X(z, \bar{z})} . .
$$

Since

$$
\begin{gathered}
\gamma(z) V\left(z^{\prime}\right) \sim i w R \log \left(z-z^{\prime}\right) V\left(z^{\prime}\right), \\
\beta(z) V\left(z^{\prime}\right) \sim \frac{-i \bar{v}}{z-z^{\prime}} V\left(z^{\prime}\right),
\end{gathered}
$$

the vertex operator carries $\left(\beta_{0}, \bar{\beta}_{0}\right)$ eigenvalues of $(-i \bar{v},-i v)$ and winding number $w$. Thus, according to (3.18), the energy $\epsilon$ and the longitudinal momentum $n / R$ of the tachyon state are given by

$$
\epsilon=v+\bar{v}+\frac{1}{2 \alpha_{\mathrm{eff}}^{\prime}} w R, \quad \frac{n}{R}=\bar{v}-v
$$

Let us evaluate the 4-point amplitude on the sphere, $\left\langle V_{1}\left(z_{1}\right) V_{2}\left(z_{2}\right) V_{3}\left(z_{3}\right) V_{4}\left(z_{4}\right)\right\rangle$, by performing the functional integral with the Lagrangian (3.8). The extremum of the functional integral is given by

$$
\gamma(z)=i \sum_{a=1}^{4} w_{a} R \log \left(z-z_{a}\right), \quad \beta(z)=-i \sum_{a=1}^{4} \frac{\bar{v}_{a}}{z-z_{a}} .
$$


For closed string amplitudes, the winding number has to be conserved (the winding number is not conserved when one considers worldsheet with boundaries), such that $\Sigma_{a} w_{a}=0$. Since the action is free, we can evaluate the amplitude by substituting (4.4) back into the integrand [as is always the case with the Gaussian integral; the same result is obtained by substituting the extremal value (4.4) into the product of the vertex operators $V_{1} V_{2} V_{3} V_{4}$ alone and by taking its square root]. The amplitude is given by

$$
\begin{aligned}
& \left\langle V_{1}\left(z_{1}\right) V_{2}\left(z_{2}\right) V_{3}\left(z_{3}\right) V_{4}\left(z_{4}\right)\right\rangle \\
& \quad=\sqrt{w_{1} \cdots w_{4}} \prod_{a \neq b}\left(z_{a}-z_{b}\right)^{-\bar{v}_{a} w_{b} R}\left(\bar{z}_{a}-\bar{z}_{b}\right)^{-v_{a} w_{b} R}\left|z_{a}-z_{b}\right|^{\left(\alpha_{\mathrm{eff}}^{\prime} / 2\right) k_{a} k_{b}} \\
& =\sqrt{w_{1} \cdots w_{4}} \prod_{a<b}\left|z_{a}-z_{b}\right|^{-\left(\epsilon_{a}+\epsilon_{b}\right)\left(w_{a}+w_{b}\right) R+\left(R^{2} / 2 \alpha_{\mathrm{eff}}^{\prime}\right)\left(w_{a}+w_{b}\right)^{2}+\left(\alpha_{\mathrm{eff}}^{\prime} / 2\right)\left(k_{a}+k_{b}\right)^{2}-4} .
\end{aligned}
$$

Here we used the on-shell condition for the tachyon,

$$
\begin{gathered}
\epsilon_{a}=\frac{w_{a} R}{2 \alpha_{\mathrm{eff}}^{\prime}}+\frac{\alpha_{\mathrm{eff}}^{\prime} k_{a}^{2}}{2 w_{a} R}-\frac{2}{w_{a} R}, \\
v_{a}=\bar{v}_{a} \quad(a=1, \ldots, 4) .
\end{gathered}
$$

It is a good test of our formalism to compute the same correlation function using the standard closed string theory and then take the NCOS limit (3.2). One can verify that (4.5) is reproduced in the limit. The tachyon scattering amplitude is then given by

$$
\mathcal{A}=i g^{4} C_{\text {sphere }} \int d^{2} z\left\langle V_{1}(0) V_{2}(z) V_{3}(1) V_{4}(\infty)\right\rangle .
$$

Here $g$ is the closed string coupling constant and $C_{\text {sphere }}$ is the normalization constant that normalizes the path integral of the string when the topology of the worldsheet is the sphere. The normalization constant can be found by unitarity. Namely, the amplitude in (4.7) has poles associated with intermediate closed string states and a straightforward application of the optical theorem determines it. Therefore, by repeating the analysis in, for example, Sec. 6.6 of Ref. 14, we find

$$
C_{\text {sphere }}=\frac{2 \pi}{g^{2} R}
$$

This means that even though the theory is defined in the $g_{s} \rightarrow \infty$ limit, that the closed string theory has a sensible perturbation expansion in powers of $g$. This is consistent with the observation made in Ref. 4 regarding closed string loop diagrams in NCOS.

The amplitude given by (4.5) and (4.7) is very similar to the familiar Virasoro-Shapiro amplitude. It has poles in the energies in the intermediate channels, and they are located at

$$
\epsilon_{a}+\epsilon_{b}=\frac{\left(w_{a}+w_{b}\right) R}{2 \alpha_{\mathrm{eff}}^{\prime}}+\frac{\alpha_{\mathrm{eff}}^{\prime}\left(k_{a}+k_{b}\right)^{2}}{2\left(w_{a}+w_{b}\right) R}+\frac{2 n-2}{\left(w_{a}+w_{b}\right) R} \quad(n=0,1,2, \ldots) .
$$

This is precisely the closed string spectrum of NRCS, as required by unitarity. From (4.7) we can also see that NRCS exhibits a different behavior of high energy, fixed-angle scattering amplitudes. Since the mass-shell condition of the strings is nonrelativistic, the dependence of the amplitude on energy $E$ is $\mathcal{A} \sim e^{-E}$, as opposed to the conventional dependence $\mathcal{A} \sim e^{-E^{2}}$.

Although there are no physical states in the sector with 0 -winding number and in particular no graviton in the spectrum, there is an instantaneous Newtonian potential between winding strings. 
To see this, let us consider the process in which the winding number is not exchanged among strings, i.e., $w_{1}+w_{3}=0$ and $w_{2}+w_{4}=0$. In this case, the correlation function (4.5) becomes

$$
\begin{aligned}
\left\langle V_{1}\left(z_{1}\right)\right. & \left.V_{2}\left(z_{2}\right) V_{3}\left(z_{3}\right) V_{4}\left(z_{4}\right)\right\rangle \\
= & w_{1} w_{2}\left(\left|z_{1}-z_{3}\right|\left|z_{2}-z_{4}\right|\right)^{\alpha_{\mathrm{eff}}^{\prime}\left(k_{1}+k_{3}\right)^{2}-4} \\
& \times\left(\left|z_{1}-z_{2}\right|\left|z_{3}-z_{4}\right|\right)^{-\left(\epsilon_{1}+\epsilon_{2}\right)\left(w_{1}+w_{2}\right) R+\left(R^{2} / 2 \alpha_{\mathrm{eff}}^{\prime}\right)\left(w_{1}+w_{2}\right)^{2}+\left(\alpha_{\mathrm{eff}}^{\prime} / 2\right)\left(k_{1}+k_{2}\right)^{2}-4} \\
& \times\left(\left|z_{1}-z_{4}\right|\left|z_{2}-z_{3}\right|\right)^{-\left(\epsilon_{1}+\epsilon_{4}\right)\left(w_{1}-w_{2}\right) R+\left(R^{2} / 2 \alpha_{\mathrm{eff}}^{\prime}\right)\left(w_{1}-w_{2}\right)^{2}+\left(\alpha_{\mathrm{eff}}^{\prime} / 2\right)\left(k_{1}+k_{4}\right)^{2}-4} .
\end{aligned}
$$

Since the winding number along the $\left(k_{1}+k_{3}\right)$-channel is 0 , no physical states are propagating in this channel. Nevertheless, after doing the $z$-integral in (4.7), one finds that there are contributions from the exchange of off-shell states in the 0 -winding number sector. In particular, the leading long-range contribution to the 4-point amplitude contains $\sim\left(k_{1}+k_{3}\right)^{-2}$, corresponding to the Newtonian potential. Thus, even though the theory contains no gravitons, there is an instantaneous gravitational force between winding strings.

\section{LOOP AMPLITUDES}

In this section, we will compute the one-loop free energy at finite temperature and one-loop corrections to $\mathrm{N}$-point functions of winding states. We will also examine the general structure of higher loop amplitudes and demonstrate that there is a sensible perturbative expansion of NRCS.

On a genus- $g$ surface, the $\beta$-field [the $(1,0)$-form we introduced in Sec. III as a Lagrangian multiplier] has $g$ zero modes. If we were quantizing the bosonic ghost system, we would introduce delta-functions in the path integral to absorb these zero modes. However, one can show that the rules of the NRCS perturbation theory deduced from the factorization conditions do not call for these delta-functions. Thus one may naively think that zero mode integrals are divergent in NRCS. This would be similar to the problem in DLCQ of field theory, ${ }^{16,17}$ where integrals over states carrying zero longitudinal momentum pose difficulties in evaluating loop amplitudes. ${ }^{18}$

It turns out that, whenever we evaluate physical observables such as the temperature dependent part of the free energy and scattering amplitudes of closed strings with nonzero winding numbers, the amplitudes contain terms of stringy nature which depend on all the $g$ zero modes of $\beta$, so that the zero-mode integrals are convergent. It is easy to understand where these terms come from; they appear because $\beta$ is a Lagrange multiplier which constrains $\gamma$ to be a holomorphic map from the worldsheet to the $(1+1)$-dimensional part of the target space. If vertex operators for winding states are inserted on the worldsheet, a holomorphic map $\gamma$, if it exists, has to be a nontrivial one since the image of the worldsheet has to wind around each of the vertex operators. As we will show below, a nontrivial holomorphic map from the worldsheet to the $(1+1)$-dimensional part of the target space, which is a cylinder, exists only in a subspace of codimension $g$ of the moduli space of a genus- $g$ Rieman surface. The integral over the $g$ zero modes of $\beta$ gives a delta-function which exactly picks up the subspace where the holomorphic maps exist.

On the other hand, if we consider amplitudes which do not contain winding strings, such as the vacuum amplitude at zero temperature, then the zero-mode integral gives a divergence. In this case, $\gamma$ can be a trivial map which maps the worldsheet to a point in the target space. Such a map exists everywhere on the moduli space of the worldsheet, and therefore the worldsheet amplitude is independent of the $g$ zero modes of $\beta$. The integral over these zero modes is then flatly divergent. If one traces through the NCOS limit in Sec. III, one finds that it is exactly the type of divergence that was pointed out in Ref. 18. Fortunately all the physical states in NRCS have a nonzero winding number, and these divergent amplitudes have no physical meaning and can be safely ignored.

We will demonstrate these points by computing one-loop amplitudes and show how they are generalized to higher loops. 


\section{A. Free energy}

The one-loop free energy at temperature $T$ is evaluated by performing a Euclidean rotation of the target space-time coordinate and periodically identifying $X^{0} \sim X^{0}+T^{-1}$. The path integral then involves a sum over maps $(\gamma, \bar{\gamma})$ from the worldsheet torus of modulus $\tau$ to the target space torus of periods $\left(T^{-1}, 2 \pi R\right)$.

The zero-mode dependence of the free energy can be computed by performing the path integral over the maps from the worldsheet to space-time. Thus we write

$$
\gamma=\left(2 \pi n R+i \frac{m}{T}\right) \frac{\sigma^{0}}{2 \pi}+\left(2 \pi w R+i \frac{s}{T}\right) \frac{\sigma^{1}}{2 \pi}+(\text { periodic }),
$$

where $0 \leqslant \sigma^{0}, \sigma^{1}<2 \pi$ and $(n, m, w, s)$ are integers labeling the different winding sectors. For this $\gamma$,

$$
\bar{\partial} \gamma=\frac{i}{4 \pi \operatorname{Im} \tau}\left[2 \pi n R+i \frac{m}{T}-\tau\left(2 \pi w R+i \frac{s}{T}\right)\right]+\bar{\partial} \text { (periodic) }
$$

On the other hand, $\beta$ can be written as $\beta=\beta_{0}+\partial$ (periodic), where $\beta_{0}$ is the zero mode. The worldsheet action depends on $\beta_{0}$ as

$$
S=i \beta_{0}\left[2 \pi n R+i \frac{m}{T}-\tau\left(2 \pi w R+i \frac{s}{T}\right)\right]+\cdots .
$$

Thus the integral over $\beta_{0}$ gives a delta-function which fixes the worldsheet modulus $\tau$ at

$$
\tau=\frac{2 \pi n R+i m \frac{1}{T}}{2 \pi w R+i s \frac{1}{T}} .
$$

Thus the $\tau$-integral becomes a sum over these special points on the worldsheet moduli space. These are the points at which there are holomorphic maps from the worldsheet to the target space.

The one-loop free energy is obtained by a sum over the integers $(n, m, w, s)$ such that $\tau$ is in the fundamental domain of the moduli space. To do the summation, it is convenient to use the trick invented in Ref. 19 to trade the sum over $s$ for the sum over copies of the fundamental domain. If $(m, s) \neq(0,0)$, there is an $S L(2, Z)$ transformation which sends $(m, s)$ to $(m, 0)$ with $m>0$, and it also maps the fundamental domain of $\tau$ into the strip, $|\operatorname{Re} \tau| \leqslant 1 / 2$, in the upper half-plane $\operatorname{Im} \tau \geqslant 0$. The sum over $s$ covers the strip exactly once by copies of the fundamental domain. On the other hand, the $(m, s)=(0,0)$ term is independent of the temperature $T$ and corresponds to the zero temperature vacuum energy. We will ignore this contribution since it has no physical meanings in NRCS and it vanishes in supersymmetric theories anyway. Thus we have

$$
\tau=\frac{2 \pi n R+i m \frac{1}{T}}{2 \pi w R},
$$

and we sum over integers $(n, m, w)$. Since $m>0$ and $\tau$ must be in the strip in the upper half-plane, we require $w>0$ and $|n| \leqslant w / 2$ ( $n$ at the boundary $n= \pm w / 2$ is counted with a factor 1/2).

We can now evaluate the path integral over $\gamma$ and $\bar{\gamma}$. The zero mode piece is obtained by evaluating the instanton action. Therefore, substituting (5.1) (with $s=0)$ into the action (3.8) and evaluating it at the points (5.5) of the moduli space, we find that the zero mode part of the action is 


$$
S=\int d^{2} z\left(\frac{1}{8 \pi \alpha_{\mathrm{eff}}^{\prime}} \partial \gamma \bar{\partial} \bar{\gamma}+\cdots\right)=\frac{m w R}{2 \alpha_{\mathrm{eff}}^{\prime} T}+\cdots
$$

As usual, the contribution from the nonzero modes of $(\beta, \gamma)$ is canceled by the determinant of the $(b, c)$ ghost system. Therefore, the one-loop contribution to the free energy takes the form

$$
F(T)=-\sum_{n, m, w} \frac{T}{w m} \sum_{h, \bar{h}} D(h, \bar{h}) \exp \left[-\frac{m}{T}\left(\frac{w R}{2 \alpha_{\mathrm{eff}}^{\prime}}+\frac{h+\bar{h}}{w R}\right)+2 \pi i \frac{n}{w}(h-\bar{h})\right] .
$$

This is obtained by evaluating the partition function of the worldsheet theory at the special points (5.5) on the moduli space. Here $(h, \bar{h})$ are the conformal weights coming from the transverse excitations of the string and $D(h, \bar{h})$ is their multiplicity. To simplify the expression in (5.7), we have included in $h$ the contribution from the transverse momenta $k$. Thus, in comparison with the notion in Sec. III, $h$ and $\bar{h}$ are defined as

$$
h=\frac{\alpha_{\text {eff }}^{\prime}}{4} k^{2}+N, \quad \bar{h}=\frac{\alpha_{\text {eff }}^{\prime}}{4} k^{2}+\bar{N} .
$$

The factor $-T / w m$ in (5.7) is determined as follows. The $\beta_{0}$ integral with the action (5.3) gives a factor $(2 \pi w R)^{-2}$ times the delta-function for $\tau$ [we set $s=0$ in (5.3)]. The measure for the $\tau$-integral contains the factor

$$
\frac{1}{\operatorname{Im} \tau}=\frac{2 \pi w R T}{m}
$$

The zero-mode integral of $\gamma$ gives the volume $2 \pi R / T$ of the target space. Finally, the definition of the one-loop free energy is $F=-T Z(T)$, where $Z(T)$ is the one-loop vacuum amplitude at temperature $T$. Combining these factors together, we obtain

$$
\frac{1}{(2 \pi w R)^{2}} \frac{2 \pi w R T}{m} \frac{2 \pi R}{T}(-T)=-\frac{T}{w m}
$$

as in (5.7).

The sum over $n$ in $|n| \leqslant w / 2$ gives the constraint $h-\bar{h} \equiv 0(\bmod w)$, which we recognize as the level matching condition. After summing over $m$, the free energy given by (5.7) becomes

$$
F(T)=T \sum_{w=1}^{\infty} \sum_{h, \bar{h}} D(h, \bar{h}) \log \left(1-e^{E(w, h, \bar{h}) / T}\right) .
$$

This is the conventional expression for the one-loop free energy of quantum field theory. Here

$$
E(w, h, \bar{h})=\frac{w R}{2 \alpha_{\mathrm{eff}}^{\prime}}+\frac{h+\bar{h}}{w R}=\frac{w R}{2 \alpha_{\mathrm{eff}}^{\prime}}+\frac{\alpha_{\mathrm{eff}}^{\prime} k^{2}}{2 w R}+\frac{N+\bar{N}}{w R}
$$

With the level matching condition for $(h, \bar{h})$, the expression for $E(w, h, \bar{h})$ precisely agrees the energy spectrum of closed strings in NRCS computed in Sec. III, with the correct multiplicity factor.

The computation of the free energy described here is similar to the one for string in $A d S_{3}$ discussed in Ref. 12. In that case, the one-loop amplitude has poles exactly at the special points in (5.4), and they are due to the presence of long strings winding near the boundary of $A d S_{3}$. Here we have delta-functions at these points and they correspond to the closed strings winding in the $x^{1}$ direction. 
We now study the high temperature behavior of the free energy. It is clear that, for a fixed winding number $w$, that the free energy is convergent for any temperature $T$. However, there might be a divergence when one performs the sum over winding modes. To see whether the sum over $w$ gives a divergence, we use the Cardy's formula,

$$
\sum_{h, \bar{h}} D(h, \bar{h}) \exp (2 \pi i \tau h-2 \pi i \bar{\tau} \bar{h}) \sim \exp \left[\frac{2 \pi i c}{24}\left(\frac{1}{\tau} \frac{1}{\bar{\tau}}\right)\right],
$$

for $\operatorname{Im} \tau \rightarrow 0$. Here $c$ is the central charge of the rest of the system, and $c=12$ for a Type II superstring. Therefore, the high temperature behavior of NRCS is given by

$$
\sum_{|n| \leqslant w / 2} \sum_{h \bar{h}} D(h, \bar{h}) \exp \left[-\frac{m}{T}\left(\frac{w R}{2 \alpha_{\mathrm{eff}}^{\prime}}+\frac{h+\bar{h}}{w R}\right)+2 \pi i \frac{n}{w}(h-\bar{h})\right] \sim \exp \left[2 \pi w R\left(\frac{\pi c T}{6 m}-\frac{m}{4 \pi \alpha_{\mathrm{eff}}^{\prime} T}\right)\right],
$$

for large $w$. Therefore, the sum over winding states will be divergent whenever $T \geqslant T_{\mathrm{H}}$, where

$$
T_{\mathrm{H}}=\frac{1}{2 \pi} \sqrt{\frac{6}{\alpha_{\mathrm{eff}}^{\prime} c}} .
$$

This gives the Hagedorn temperature of NRCS. For Type II NRCS we find that $T_{\mathrm{H}}$ $=1 / 2 \pi \sqrt{2 \alpha_{\mathrm{eff}}^{\prime}}$. It coincides with the location of the Hagedorn transition of NCOS studied in Refs. 11, 20. Just like in conventional string theory, the Hagedorn temperature $T_{\mathrm{H}}$ is the temperature at which the tachyonic mode which appears in the spectrum (5.12) due to the finite temperature GSO projection becomes massless. It would be interesting to study the behavior of the closed strings of NRCS above the Hagedorn temperature. In NRCS, the breakdown of the thermal ensemble may not occur unlike for relativistic closed string theories since there is no graviton and the Hamiltonian is positive definite. However, there can be a Jeans instability.

We have demonstrated explicitly that the $\beta$ zero-mode integral is convergent when one computes the one-loop free energy. As a result of the $\beta$ zero-mode integral, the integral over $\tau$ is localized to a sum over the special points in the moduli space of the worldsheet torus where there is a holomorphic map from the worldsheet to the target space torus. It is straightforward to generalize this observation to higher loops. A simple computation shows that a map from a genus $g$ worldsheet to the target space torus exists only on a $(2 g-3)$-dimensional subspace. Such a holomorphic map exists whenever the following condition on the worldsheet period matrix $\Omega_{i j}(i, j=1, \ldots, g)$ is satisfied:

$$
G_{i}(\Omega)=\sum_{j=1}^{g} \Omega_{i j}\left(2 \pi w^{j} R+i \frac{s^{j}}{T}\right)+2 \pi n_{i} R+i \frac{m_{i}}{T}=0,
$$

for some integers $\left(n_{i}, m_{i}, w^{i}, s^{i}\right)$. This generalizes (5.4) for $g=1$. On a genus- $g$ surface, $\beta$ has $g$ linearly independent zero modes, and their integrals give delta-functions imposing the constraints $G_{i}=0(i=1, \ldots, g)$.

\section{B. $N$-point amplitudes}

Here we analyze the effects of the $\beta$ zero-mode integrals on the scattering amplitudes. We will discuss the $\mathrm{N}$-point tachyon amplitudes for simplicity, but a generalization to amplitudes involving arbitrary external states is straightforward. As in the case of the tree amplitudes discussed in the last section, the computation of $\left\langle\pi_{i=1}^{n} e^{i \bar{v}_{a} \gamma\left(u_{a}\right)+v_{a} \bar{\gamma}\left(\bar{x}_{a}\right)}\right\rangle$ requires finding $(\beta, \gamma)$ which are holomorphic away from $u_{a}$ 's and behave near $z=u_{a}$ as 


$$
\begin{aligned}
& \gamma(z) \sim i w_{a} R \log \left(z-u_{a}\right), \\
& \beta(z) \sim \frac{-i \bar{v}_{a}}{z-u_{a}} \quad\left(z \rightarrow u_{a}\right),
\end{aligned}
$$

where $w_{a}$ is the winding number for the $i$-th external state. The functional integral is nonzero only when such $\beta$ and $\gamma$ exist. As for $\beta(z)$, there is always a meromophic one-form given by

$$
\beta(z)=-i \sum_{a=1}^{N} \bar{v}_{a} \partial_{z} \log \vartheta_{1}\left(z-u_{a}\right)+\text { const }
$$

where $\vartheta_{1}(z)$ is the elliptic theta function. This $\beta$ is single-valued on the worldsheet torus as far as momentum is conserved, $\Sigma_{a} v_{a}=0$. On the other hand, $\gamma(z)$ obeying (5.17) does not always exist. To see this, let us try

$$
\gamma(z)=i \sum_{a=1}^{N} w_{a} R \log \vartheta_{1}\left(z-u_{a}\right)+c z
$$

for some constant $c$. Due to the quasi-periodicity of the elliptic function, we find

$$
\begin{gathered}
\gamma(z+2 \pi)=\gamma(z)+2 \pi c \\
\gamma(z+2 \pi r)=\gamma(z)+2 \pi\left(-R \sum_{a=1}^{N} w_{a} u_{a}+c \tau\right),
\end{gathered}
$$

where we assumed that the winding number is conserved, $\Sigma_{a} w_{a}=0$. By requiring that $\gamma(z)$ is periodic modulo the target space periodicity $\gamma \sim \gamma+2 \pi R$, we find that $c$ must be of the form $c$ $=m R$ for some integer $m$ and

$$
\sum_{a=1}^{N} w_{a} u_{a}=n+m \tau,
$$

for some integer $n$. This gives a condition on the locations of the $N$ points. Thus we find that, rather than being divergent, the integral over the zero mode of $\beta$ imposes the condition (5.21) on the locations of the vertex operators on the worldsheet.

High loops

It is straightforward to generalize this result to higher loops. On a genus- $g$ worldsheet, the holomorphic map $\gamma(z)$ winding $w_{a}$-times at $z=u_{a}$ should be of the form

$$
\gamma(z)=i \sum_{a=1}^{N} w_{a} R \log E\left(z, u_{a}\right)+\sum_{i=1}^{g} c^{i} \int^{z} \omega_{i},
$$

for some constants $c_{i}$, where $\omega_{i}$ are holomorphic one-forms, and $E(z, w)$ is the prime form, a $(-1 / 2)$-differential with respect to $z$ and $w$ that vanishes linearly on the diagonal $z=w$ only (see, for example, Sec. IIIb.1 of Ref. 21). The periodicity of $\gamma$ in the $\alpha$-cycles of the worldsheet requires that $c^{i}$ must be of the form $c^{i}=2 \pi m^{i} R$ for some integers $m^{i}$, and the periodicity around the $\beta$-cycles requires

$$
G_{i}=\sum_{a=1}^{N} w_{a}{ }^{u_{a}} \omega_{i}-\left(n_{i}+\sum_{j=1}^{g} \Omega_{i j} m^{j}\right)=0,
$$

for some integers $n_{i}$. This imposes $g$ conditions on the $(3 g-3+N)$-dimensional moduli space of the genus- $g$ worldsheet with $N$ points. To verify that the $g$ conditions $G_{i}=0$ are independent of 
each other and pick up a codimension $g$ subspace, we need to compute their partial derivatives with respect to the worldsheet moduli $y_{I}(I=1, \ldots, 3 g-3)$ and the vertex operator locations $u_{a}$. They are given by

$$
\begin{gathered}
\frac{\partial G_{i}}{\partial u_{a}}=\omega^{i}\left(u_{a}\right) w_{a}, \\
\frac{\partial G_{i}}{\partial y_{I}}=\int d^{2} z \omega_{i}(z) \eta^{I}(z, \bar{z})\left(\sum_{a=1}^{N} w_{a} \partial_{z} \log E\left(z, u_{a}\right)-2 \pi i \sum_{j=1}^{g} m^{j} \omega_{j}(z)\right),
\end{gathered}
$$

where $\eta^{I}$ are the Beltrami differentials associated to the moduli $y_{I}$. Note that the partial derivatives of $G_{i}$ are all of the form, $\int d^{2} z \omega_{i}(z) \nu(z, \bar{z})$ for some differential $\nu$. Since

$$
\operatorname{det}_{i, j=1, \cdots g}\left(\omega_{i}\left(z_{j}\right)\right) \neq 0,
$$

for generic $g$ points $z_{i}$, it is clear that the rank of the $(3 g-3+N) \times g$ matrix $\left(\partial_{u_{a}} G_{i}, \partial_{y_{I}} G_{i}\right)$ is generically $g$. Thus the integral over the $g$ zero modes of $\beta$ exactly pick up the subspace of the moduli space where the holomorphic map $\gamma(z)$ exists.

\section{RELATION TO DLCQ}

In this section we show that the NRCS limit we have studied is related by T-duality to the DLCQ limit of string theory. This follows by performing T-duality along the circle of radius $R$ where the $B$-field lies. After T-duality, we get string theory without any background $B$-field, with a metric

$$
g_{\mu \nu}=\left(\begin{array}{cc}
-1+\left(2 \pi \alpha^{\prime} B\right)^{2} & 2 \pi \alpha^{\prime} B \\
2 \pi \alpha^{\prime} B & 1
\end{array}\right),
$$

and where the radius of the circle is $\alpha^{\prime} / R$. In the NRCS limit the metric is given by

$$
d s^{2}=-\frac{\alpha^{\prime}}{\alpha_{\mathrm{eff}}^{\prime}}\left(d x^{0}\right)^{2}+2 d x^{0} d x^{1}+\left(d x^{1}\right)^{2} .
$$

We now rescale coordinates such that $x^{1} \rightarrow\left(\alpha^{\prime} / \alpha_{\mathrm{eff}}^{\prime}\right) x^{1}$. In the NRCS $\left(\alpha^{\prime} \rightarrow 0\right)$ limit the metric in these coordinates is

$$
\frac{1}{\alpha^{\prime}} d s^{2}=\frac{1}{\alpha_{\mathrm{eff}}^{\prime}}\left[-\left(d x^{0}\right)^{2}+2 d x^{0} d x^{1}\right]
$$

and the periodicity of the compact direction is given by

$$
x^{1} \sim x^{1}+2 \pi \frac{\alpha_{\mathrm{eff}}^{\prime}}{R} .
$$

Since in this limit the $x^{1}$ coordinate is light-like, we see that DLCQ of closed string theory with string scale $\alpha_{\text {eff }}^{\prime}$ and null radius $\alpha_{\text {eff }}^{\prime} / R$ is T-dual to NRCS.

Because of the relation between NRCS and DLCQ, the formalism developed in this paper gives a useful description of DLCQ closed string theory also. In Refs. 22, 23, loop amplitudes of DLCQ closed string theory were studied to address the issue of divergence due to the longitudinal zero modes. ${ }^{16-18}$ In particular, it was found in Ref. 22 that one-loop scattering amplitudes, when external strings carry nonzero longitudinal momenta, have finite DLCQ limits and that the positions of the vertex operators are constrained in the limit. These constraints can be viewed as the T-dual of (5.21) in NRCS. The description of NRCS developed here does not involve the process 
of taking the NCOS limit, and thus loop amplitudes are manifestly finite. In fact, the one-loop observation in Ref. 22 has a straightforward generalization to higher loops, as we saw in (5.23). Evidently, unlike in ordinary field theories, the longitudinal zero modes do not cause a problem in DLCQ closed string theory. In the case of Type IIA string theory, this is related, via the hypothetical 11-dimensional Lorentz invariance of M theory, to the existence of the smooth DLCQ limit of M Theory described by the finite $N$ Matrix Theory. ${ }^{24-26}$

For closed strings, the relation between NRCS and DLCQ provides another way to understand the origin of the nonrelativistic dispersion relation. The nonrelativistic limit described in Sec. II, however, is much broader and includes cases that are not related to DLCQ, as we will see in the next section.

\section{GALILEAN THEORIES}

In this section we find new theories whose excitations satisfy a nonrelativistic dispersion relation. The light degrees of freedom that survive the low energy limit are light-branes (these theories do not have background branes, unlike the theories discussed in Ref. 8). Depending on which background gauge field one tunes to its critical value, different brane states are light while the rest of the states in M Theory decouple.

We will first consider M Theory limits where the light degrees of freedom are membranes and five-branes. We will call these theories GM (Galilean membrane) and GF (Galilean five-brane), respectively. The first one is obtained by tuning to the critical value the background three-form and the second one by turning on the background six-form of M Theory. The low energy limit is taken such that the terms in the world volume action depending on the transverse coordinates to the background remain finite in the limit. Just as for NRCS, the spectrum is modified if the brane directions are compactified, otherwise there are no finite energy physical excitations surviving the limit.

Therefore, the low energy limit leading to GM is given by (throughout the rest of the paper we will have in mind compactification on tori; it is straightforward to generalize the decoupling limits when branes wrap curved geometries)

$$
\begin{gathered}
g_{\mu \nu}=\eta_{\mu \nu} \quad \mu, \nu=0,1,2, \\
g_{i j}=\frac{l_{p}^{3}}{l_{\text {eff }}^{3}} \delta_{i j} \quad i, j=3, \ldots, 10, \\
C_{012}=T_{\mathrm{M} 2}-T_{\text {eff }},
\end{gathered}
$$

with the eleven-dimensional Planck scale $l_{p} \rightarrow 0$ while the effective length scale $l_{\text {eff }}$ is kept finite. Here $T_{\mathrm{M} 2}=1 / 4 \pi^{2} l_{p}^{3}$ is the membrane tension and $T_{\text {eff }}=1 / 4 \pi^{2} l_{\text {eff }}^{3}$ is the finite effective tension of the light membranes that survive the limit. Note that GM has no coupling constant and it contains a unique dimensionful parameter $l_{\text {eff }}$. This is reminiscent of some of the properties of elevendimensional M Theory.

The decoupling limit giving rise to GF is given by

$$
\begin{gathered}
g_{\mu \nu}=\eta_{\mu \nu}, \quad \mu, \nu=0,1, \ldots, 5, \\
g_{i j}=\frac{l_{p}^{6}}{l_{\mathrm{eff}}^{6}} \delta_{i j}, \quad i, j=6, \ldots, 10, \\
C_{012345}=T_{\mathrm{M} 5}-T_{\mathrm{eff}},
\end{gathered}
$$

with $l_{p} \rightarrow 0$ while the effective length scale $l_{\text {eff }}$ is kept finite. Here $T_{\text {eff }}$ denotes the effective tension of the light five-brane excitations. Just as GM, GF has no coupling constant and $l_{\text {eff }}$ is the unique dimensionless parameter of the theory. 
Similar theories can be obtained from light Dp-branes, which we will call GDp (Galilean D-p-branes). As we will now see, these theories are connected by dualities which are reminiscent of the dualities of the fully relativistic theories from which we obtain these Galilean theories. We first discuss the relation between supersymmetric Type IIA NRCS and the eleven-dimensional theory GM.

Here we will show that in fact the strong coupling dual to Type IIA NRCS is described by eleven-dimensional GM such that the parameters of the two theories are related to each other in a similar fashion to the usual relation in the relativistic setting.

Consider GM theory (7.1) compactified on a circle of proper radius $R$. The background critical three-form potential reduces to a NS-NS two-form potential,

$$
B_{01}=2 \pi R C_{012} .
$$

Using the usual relation of parameters between M theory and Type IIA superstring theory,

$$
R=g_{s} \sqrt{\alpha^{\prime}}, \quad l_{p}=g_{s}^{1 / 3} \sqrt{\alpha^{\prime}},
$$

we can write $R$ and $l_{p}$ in the limit (3.2) which defines NRCS, so that

$$
R=g \sqrt{\alpha_{\mathrm{eff}}^{\prime}}, \quad l_{p}=g^{1 / 3} \alpha_{\mathrm{eff}}^{\prime 1 / 6} \alpha^{1 / 3},
$$

as $\alpha^{\prime} \rightarrow 0$.

Substituting the eleven-dimensional limit (7.1) in (7.3) we see that the background NS-NS two-form potential is given by

$$
2 \pi \alpha^{\prime} B_{01}=1-\frac{l_{p}^{3}}{l_{\mathrm{eff}}^{3}}
$$

Using (7.5) and comparing with the limit defining NRCS in (3.2), we see that NRCS with coupling constant $g$ and effective string scale $\alpha_{\text {eff }}^{\prime}$ is equivalent to GM theory on a circle of radius $R$ and effective Planck scale $l_{\text {eff }}$. The parameters are related by

$$
R=g \sqrt{\alpha_{\mathrm{eff}}^{\prime}}, \quad l_{\mathrm{eff}}=g^{1 / 3} \sqrt{\alpha_{\mathrm{eff}}^{\prime}} .
$$

\section{A. GDp and GNSF theories}

In this subsection we present some generalizations to the construction we made for perturbative closed strings in a near critical NS-NS $B$-field to Dp-branes in a near critical RamondRamond $p+1$-form background. In order for the background to affect the Hamiltonian, the spatial directions of the brane have to be compactified on an orientable $p$-cycle of space-time. In this case, the winding number plays the role of electric charge in the discussion in (2.4) and again positivity of the energy allows only wrapping in one orientation.

The nonrelativistic limit that needs to be taken requires keeping finite the terms in the world volume action depending on the transverse coordinates to the brane and tuning the background field to the tension of the D-brane (this is the analog of the NRCS limit. There, we scaled $\alpha^{\prime}$ and the metric in the transverse directions to the $B$-field such that terms of the string worldsheet action depending on the transverse coordinates are kept finite). The limit is given by

$$
\begin{gathered}
g_{\mu \nu}=\eta_{\mu \nu}, \quad \mu, \nu=0,1, \ldots, p, \\
g_{i j}=\left(\frac{\alpha^{\prime}}{\alpha_{\mathrm{eff}}^{\prime}}\right)^{2} \delta_{i j}, \quad i, j=p+1, \ldots, 9, \\
g_{s}=\left(\frac{\alpha^{\prime}}{\alpha_{\mathrm{eff}}^{\prime}}\right)^{(3-p) / 2} g_{p},
\end{gathered}
$$




$$
C_{01 \cdots p}=T_{p}-T_{p}^{\mathrm{eff}},
$$

as $\alpha^{\prime} \rightarrow 0$. Here $T_{p}$ is the tension of the D-brane (which is infinite in the limit),

$$
T_{p}=\frac{1}{(2 \pi)^{p} g_{s} \alpha^{\prime(p+1) / 2}} .
$$

$T_{p}^{\text {eff }}$ is the finite scale of the nonrelativistic theory and $g_{p}$ the coupling of the theory. The effective tension is given by

$$
T_{p}^{\mathrm{eff}}=\frac{1}{(2 \pi)^{p} g_{p} \alpha_{\mathrm{eff}}^{(\prime p+1) / 2}} .
$$

In the limit (7.8) all states of string theory decouple except the light Dp-branes that have finite proper energy excitations. In particular, open and closed strings decouple from the low energy spectrum.

There is a very simple explanation for the limit we take in (7.8). Except for the presence of the background gauge field $C_{01 \ldots p},(7.8)$ is the conventional low energy limit that results in a gauge theory on a Dp-brane. ${ }^{27}$ This can be easily recognized by noting that the Yang-Mills coupling constant is given by $g_{\mathrm{YM}}^{2} \sim g_{p} \alpha_{\mathrm{eff}}^{\prime(p-3) / 2}$ and that the limit (7.8) keeps the coupling finite. Moreover, the metric for the transverse coordinates $X^{i}$ to the brane is given as in (7.8) whenever we express them in terms of the Higgs field $\Phi^{i}$ of the gauge theory as $X^{i}=\alpha^{\prime} \Phi^{i}$ and require that the metric for $\Phi^{i}$ remains finite. The low energy limit is supplemented by turning a near critical background gauge field which results in light Dp-brane fluctuations.

There is one more theory we can define by tuning a massless gauge field of string theory, namely the one where the light excitations are NS five-branes. We will call these theories GNSF (Galilean Neveu-Schwarz five-brane). Just like NRCS, these theories can be obtained as a low energy limit of the different superstring theories. This low energy limit can also be motivated by considering the low energy limit on NS five-branes which yields the little string theories. ${ }^{28}$ The nonrelativistic limit is given by

$$
\begin{gathered}
g_{\mu \nu}=\eta_{\mu \nu}, \quad \mu, \nu=0,1, \ldots, 5, \\
g_{i j}=\left(\frac{g_{s}}{G}\right)^{2} \delta_{i j}, \quad i, j=6, \ldots, 9, \\
B_{012345}=T_{5}-T_{5}^{\mathrm{eff}},
\end{gathered}
$$

as $g_{s} \rightarrow 0$ while keeping the string scale $\alpha^{\prime}$ finite in the limit. Now the effective tension of the light NS five-branes is given by

$$
T_{5}^{\mathrm{eff}}=\frac{1}{(2 \pi)^{5} G^{2} \alpha_{\mathrm{eff}}^{\prime 3}} .
$$

Thus, (7.11) defines a nonrelativistic theory GNSF of light fluctuations of NS five-branes.

We will now briefly describe the theory one obtains for different values of $p$.

\section{Zero-branes}

For GD0, one may lift the description to M Theory (for a lift to eleven dimensions of a similar limit see Ref. 8). After a suitable rescaling of energy scales in M Theory, the M Theory circle goes from a space-like circle to a light-like circle of finite radius $R=g_{0} \sqrt{\alpha_{\text {eff }}^{\prime}}$. Then, if we consider the limit (7.8) for $N$ D0-branes one obtains a DLCQ description of M Theory with eleven-dimensional Planck length $l_{p}=g_{0}^{1 / 3} \sqrt{\alpha_{\text {eff }}^{\prime}}$ in a sector with $N$ units of longitudinal momentum. 


\section{One-branes}

For $p=1$, the light states are D1-branes. The strong coupling dual of the theory of light D1-branes can be found by using Type IIB S-duality. S-duality maps the critical R-R background $C_{01}$ to a critical NS-NS background $B_{01}$. The parameters $\widetilde{g}_{s}$ and $\widetilde{\alpha}^{\prime}$ of the S-dual theory are given by

$$
\widetilde{g}_{s}=\frac{1}{g_{s}}, \quad \widetilde{\alpha}^{\prime}=\alpha^{\prime} g_{s} .
$$

Writing the limit (7.8) for $p=1$ in terms of the S-dual variables and comparing with (3.2) shows that the S-dual of GD1 is given precisely by NRCS if the parameters of the two theories are related by

$$
g=\frac{1}{\widetilde{g}}, \quad \alpha_{\mathrm{eff}}^{\prime}=\widetilde{g} \widetilde{\alpha}_{\mathrm{eff}}^{\prime} .
$$

Thus Type IIB NRCS is related to GD1 by a strong-weak coupling duality which takes the same form as the conventional Type IIB S-duality. At the end of this section we will realize S-duality of Type IIB NRCS by studying GM theory compactified on a two-torus. ${ }^{29,30}$

\section{Two-branes}

GD2 can also be lifted to an eleven-dimensional picture. The D2-branes lift to M2-branes and the parameters (the eleventh-dimensional proper radius $R$ and the Planck length $l_{p}$ ) of M Theory are related to those of GD2 by

$$
R=\frac{\alpha^{\prime}}{\sqrt{\alpha_{\mathrm{eff}}^{\prime}}} g_{2}, \quad l_{p}=g_{2}^{1 / 3} \frac{\alpha^{\prime 2 / 3}}{\alpha_{\mathrm{eff}}^{\prime 1 / 6}} .
$$

Moreover, the near critical R-R background lifts to a near critical background for the three-form of eleven-dimensional supergravity. Therefore, GD2 can be identified with GM on a transverse circle. Using the parameters in (7.1) we see that GM with an effective Planck scale $l_{\text {eff }}$ on a transverse circle of coordinate size L is GD2 with coupling $g_{2}$ and effective string scale $\alpha_{\text {eff }}^{\prime}$. The parameters are related by

$$
L=g_{2} \sqrt{\alpha_{\text {eff }}^{\prime}}, \quad l_{\text {eff }}=g_{2}^{1 / 3} \sqrt{\alpha_{\text {eff }}^{\prime}} .
$$

Thus, the relation between GD2 and GM is reminiscent of the conventional duality between Type IIA and M Theory.

\section{Three-branes}

In order to get light D3-branes one must turn on a critical RR four-form. We can analyze the strong coupling dual of GD3. In fact, GD3 is self-dual, since S-duality of the underlying string theory maps the limit (7.8) to an analogous limit but with a different coupling constant and effective string scale. Therefore, GD3 with parameters $g_{3}$ and $\alpha_{\text {eff }}^{\prime}$ is dual to GD3 with parameters $\widetilde{g}_{3}$ and $\widetilde{\alpha}_{\text {eff }}^{\prime}$. The parameters are related by

$$
\widetilde{g}_{3}=\frac{1}{g_{3}}, \quad \widetilde{\alpha}_{\mathrm{eff}}^{\prime}=g_{3} \alpha_{\mathrm{eff}}^{\prime} .
$$

At the end of this section we realize the S-duality of CD3 from an eleven-dimensional perspective. 


\section{Four-branes}

The GD4 limit can also be lifted to M Theory. The main difference here is that $g_{s} \rightarrow \infty$ in the limit. Therefore, it is required to analyze the configuration in eleven dimensions. The D4-branes lift to M5-branes wrapped on the M Theory circle. The proper size of the circle and the corresponding eleven-dimensional Planck length are given by

$$
R=g_{4} \sqrt{\alpha_{\mathrm{eff}}^{\prime}}, \quad l_{p}=g_{4}^{1 / 3} \alpha^{\prime 1 / 3} \alpha_{\mathrm{eff}}^{\prime 1 / 6} .
$$

Since the background RR five-form lifts to a near critical six-form of eleven-dimensional supergravity, one sees that the strong coupling dual to GD4 is given by GF. Comparing the parameters in (7.18) with those that define GF (7.2) one finds that the parameters of the two theories are related by

$$
R=g_{4} \sqrt{\alpha_{\mathrm{eff}}^{\prime}}, \quad l_{\mathrm{eff}}=g_{4}^{1 / 3} \sqrt{\alpha_{\mathrm{eff}}^{\prime}} .
$$

\section{Five-branes}

We now consider the OD5 theory. For $p=5$, one also has to perform S-duality since in the decoupling limit $g_{s} \rightarrow \infty$. S-duality maps the D5-branes to NS five-branes. Moreover, the critical RR field gets mapped to a critical NS-NS electric field for the NS five-branes. Thus we are led to studying NS five-branes in a critical field. Type IIB S-duality maps the limit (7.8) for $p=5$ to a theory with string scale and string coupling given by

$$
\widetilde{\alpha}^{\prime}=g_{s} \alpha^{\prime}=g_{5} \alpha_{\mathrm{eff}}^{\prime}, \quad \widetilde{g}_{s}=\frac{1}{g_{s}}=\frac{\alpha^{\prime}}{\alpha_{\mathrm{eff}}^{\prime}} \frac{1}{g_{5}} .
$$

Note that the string scale of the S-dual theory is finite while the string coupling vanishes. This is precisely the limit that defines Type IIB GNSF (7.11). The parameters of GD5 $\left(g_{5}, \alpha_{\text {eff }}^{\prime}\right)$ are related to those of $\operatorname{GNSF}\left(G, \widetilde{\alpha}^{\prime}\right)$ by

$$
\alpha_{\mathrm{eff}}^{\prime}=G \widetilde{\alpha}^{\prime}, \quad G=\frac{1}{g_{5}} .
$$

In the limit that defines GD5, apart from having low energy D5-brane excitations, there are also finite energy D1-string excitations. These are identified in the Type IIB GNSF theory with strings of tension $\widetilde{\alpha}^{\prime-1}$ fluctuating inside the five-branes.

\section{Type IIA Neveu-Schwarz five-branes}

Type IIA GNSF has an interesting strong coupling dual. The limit in (7.11) can be realized by considering the decoupled theory of light fluctuating five-branes of $\mathrm{M}$ Theory on a transverse circle of proper size $R$. The near critical six-form background of eleven-dimensional supergravity becomes a near critical RR six-form of Type IIA string theory and the M5-brane becomes a NS five-brane on a transverse circle of proper radius $R$. The parameters of the two theories are related by

$$
R=g_{s} \sqrt{\alpha^{\prime}}, \quad l_{p}=g_{s}^{1 / 3} \sqrt{\alpha^{\prime}} .
$$

By comparing the scaling limit (7.2) with (7.11) and using (7.22) one finds the following relation between the effective length scales of the two theories,

$$
l_{\mathrm{eff}}=G^{1 / 3} \sqrt{\alpha_{\mathrm{eff}}^{\prime}} .
$$

Moreover, the NS five-branes now sit at a point in the transverse circle, whose coordinate length is given by 


$$
L=G \sqrt{\alpha^{\prime}} .
$$

We see that the Galilean theories we have found sit on the same moduli space and satisfy many of the properties of the parent theories from which we define them by taking a low energy limit. The reduced number of degrees of freedom that these theories have can be an interesting avenue in which to study in a simplified setting M Theory dualities.

\section{S-duality of Type IIB NRCS from GM}

We first notice that Type IIB NRCS can be obtained by considering eleven-dimensional GM theory compactified on a two-torus, where one circle is along the membrane and the other is transverse to it, and then shrinking the torus.

Let us consider GM theory compactified on a rectangular torus of coordinate size radii $R_{1}$ and $R_{2}$. We will take the circle of radius $R_{1}$ to be along the background three-form of supergravity and the circle of radius $R_{2}$ to be transverse to it. If we reduce GM on $R_{1}$ one gets Type IIA NRCS. The string coupling is given by

$$
g_{s}=\frac{R_{1}}{\sqrt{\alpha^{\prime}}}
$$

We now perform T-duality along the circle of radius $R_{2}$. This maps the limit to a Type IIB set-up. T-duality inverts the proper size of the circle one T-duals along and changes the dilaton in the conventional fashion. The new string coupling is given by

$$
g_{s}^{\prime}=\frac{R_{1}}{R_{2}} \sqrt{\frac{\alpha_{\text {eff }}^{\prime}}{\alpha^{\prime}}}
$$

Therefore, comparing with (3.2) we see that this compactification of GM leads to Type IIB NRCS with coupling constant

$$
g=\frac{R_{1}}{R_{2}}
$$

compactified on a transverse circle of coordinate size $\alpha_{\mathrm{eff}}^{\prime} / R_{2}$. Therefore, one gets Type IIB NRCS from GM in the limit that the coordinate area of the torus vanishes at fixed ratio $R_{1} / R_{2}$.

However, one could have chosen to reduce GM on the circle of coordinate radius $R_{2}$. As we showed, this leads to CD2 theory on a parallel circle of coordinate size $R_{1}$. The Type IIA coupling is given by

$$
\widetilde{g}_{s}=\frac{\sqrt{\widetilde{\alpha}^{\prime}}}{\widetilde{\alpha}_{\text {eff }}^{\prime}} R_{2}
$$

One can perform a T-duality transformation along the circle of radius $R_{1}$ such that we get a low energy limit in the Type IIB superstring where the string coupling is given by

$$
\widetilde{g}_{s}^{\prime}=\frac{\widetilde{\alpha}^{\prime}}{\widetilde{\alpha}_{\text {eff }}^{\prime}} \frac{R_{2}}{R_{1}}
$$

and the circle is of coordinate size $\widetilde{\alpha}_{\text {eff }}^{\prime} / R_{1}$. Thus, by looking at (7.8) for $p=1$ we see that one gets CD1 theory with coupling,

$$
\widetilde{g}_{1}=\frac{R_{2}}{R_{1}} .
$$


Therefore, S-duality of Type IIB NRCS can be understood from an eleven-dimensional perspective as the symmetry that exchanges the two circles when one considers GM theory compactified on a two-torus. ${ }^{29,30}$

\section{S-duality of GDS from GF}

Consider a single M5 brane compactified on a rectangular two-torus of radii $R_{1}$ and $R_{2}$ along the background directions in the decoupling limit (7.2). If we treat the circle of radius $R_{1}$ to be the one that results in GD4, then the parameters of GD4 are given by

$$
R_{1}=g_{4} \sqrt{\alpha_{\mathrm{eff}}^{\prime}}, \quad l_{\mathrm{eff}}=g_{4}^{1 / 3} \sqrt{\alpha_{\mathrm{eff}}^{\prime}} .
$$

One may perform T-duality along the circle of radius $R_{2}$. Then, we obtain the Galilean theory of light D3-branes. The string coupling after T-duality is given by

$$
g_{s}^{\prime}=g_{s} \frac{\sqrt{\alpha^{\prime}}}{R_{2}}=g_{4} \sqrt{\frac{\alpha_{\mathrm{eff}}^{\prime}}{R_{2}}}
$$

Comparing (7.32) with (7.8) for $p=3$ and using (7.31) one finds that the effective coupling of the theory of light D3-branes is

$$
g_{3}=\frac{R_{1}}{R_{2}}
$$

which is reminiscent of the relation between M Theory on a two-torus and Type IIB string theory.

If one reduces first on the circle of radius of $R_{2}$ and then performs T-duality along the circle of radius $R_{1}$ one again obtains GD3 but with the inverse coupling. Therefore, S-duality of GD3 follows from the interchange of the two circles of the two-torus in the GF realization of GD3.

\section{ACKNOWLEDGMENTS}

We would like to thank O. Bergman, J. Maldacena, T. Mehen, J. Schwarz, M. Wise, and E. Witten for useful comments. This research is supported in part by the Department of Energy (DOE) Grant No. DE-FG03-92ER40701 and the Caltech Discovery Fund.

${ }^{1}$ T. Banks, W. Fischler, S. H. Shenker, and L. Susskind, “M theory as a matrix model: A conjecture," Phys. Rev. D 55, $5112(1997)$.

${ }^{2}$ J. Maldacena, "The large $N$ limit of superconformal field theories and supergravity," Adv. Theor. Math. Phys. 2, 231 (1998).

${ }^{3}$ N. Seiberg, L. Susskind, and N. Toumbas, "Strings in background electric field, space/time noncommutativity and a new noncritical string theory," J. High Energy Phys. 6, 21 (2000).

${ }^{4}$ R. Gopakumar, J. Maldacena, S. Minwalla, and A. Strominger, "S duality and non-commutative gauge theory," J. High Energy Phys. 6, 36 (2000).

${ }^{5}$ J. Gomis and T. Mehen, "Spacetime noncommutative field theories and unitarity," hep-th/0005129.

${ }^{6}$ N. Seiberg, L. Susskind, and N. Toumbas, "Spacetime noncommutativity and causality," J. High Energy Phys. 6, 44 (2000).

${ }^{7}$ O. Aharony, J. Gomis, and T. Mehen, “'On theories with light-like noncommutativity," hep-th/0006236.

${ }^{8}$ R. Gopakumar, S. Minwalla, N. Seiberg, and A. Strominger, "OM theory in diverse dimensions," J. High Energy Phys. 8, 8 (2000).

${ }^{9}$ E. Bergshoeff, D. S. Berman, J. P. van der Schaar, and P. Sundell, "Critical fields on the M5-brane and noncommutative open strings," hep-th/0006112.

${ }^{10}$ T. Harmark, "Open branes in space-time non-commutative little string theory," hep-th/0007147.

${ }^{11}$ I. R. Klebanov and J. Maldacena, " $(1+1)$-dimensional NCOS and its $U(N)$ gauge theory dual," hep-th/0006085.

${ }^{12}$ J. Maldacena, H. Ooguri, and J. Son, "Strings in $A d S_{3}$ and the $\operatorname{SL}(2, R)$ WZW model. II: Euclidean black hole," J. Math. Phys. 42, 2961 (2001).

${ }^{13}$ M. Kato and K. Ogawa, "Covariant quantization of string based on BRS invariance,” Nucl. Phys. B 212, 443 (1983).

${ }^{14}$ J. Polchinski, String Theory (Cambridge University Press, Cambridge, 1998), Vol. I.

${ }^{15}$ T. Kugo and I. Ojima, "Manifestly covariant canonical formalism of the Yang-Mills field theories I," Prog. Theor. Phys. 60, 1869 (1978). 
${ }^{16} \mathrm{~T}$. Maskawa and K. Yamawaki, "The problem of $P^{+}=0$ mode in the null plane field theory and Dirac's method of quantization," Prog. Theor. Phys. 56, 270 (1976).

${ }^{17}$ S. Brodsky and C. Pauli, "Discretized light cone quantization: Solution to a field theory in one space one time dimensions," Phys. Rev. D 32, 2001 (1985).

${ }^{18}$ S. Hellerman and J. Polchinski, "Compactification in the lightlike limit," Phys. Rev. D 59, 125002 (1999).

${ }^{19}$ J. Polchinski, "Evaluation of the one loop string path integral," Commun. Math. Phys. 104, 37 (1986).

${ }^{20}$ S. S. Gubser, S. Gukov, I. R. Klebanov, M. Rangamani, and E. Witten, "The Hagedorn transition in non-commutative open string theory,"' hep-th/0009140.

${ }^{21}$ D. Mumford, Tata Lectures on Theta II (Birkhäuser, Basel, 1984).

${ }^{22}$ A. Bilal, "A comment on compactification of M theory on an (almost) lightlike circle," Nucl. Phys. B 521, 202 (1998); "DLCQ of M theory as the lightlike limit," Phys. Lett. B 435, 312 (1998).

${ }^{23}$ S. Uehara and S. Yamada, "On the DLCQ as a light-like limit in string theory," hep-th/0008146.

${ }^{24}$ L. Susskind, "Another conjecture about M(atrix) theory," hep-th/9704080.

${ }^{25}$ A. Sen, "D0-branes on $T^{n}$ and matrix theory," Adv. Theor. Math. Phys. 2, 51 (1998).

${ }^{26}$ N. Seiberg, "Why is the matrix model correct?," Phys. Rev. Lett. 79, 3577 (1997).

${ }^{27}$ M. Douglas, D. Kabat, P. Pouliot, and S. H. Shenker, "D-branes and short distances in string theory," Nucl. Phys. B 485, 85 (1997), hep-th/9608024.

${ }^{28}$ N. Seiberg, "Matrix description of M-theory on $T^{5}$ and $T^{5} / Z_{2}$," Phys. Lett. B 408, 98 (1997).

${ }^{29}$ P. S. Aspinwall, "Some relationships between dualities in string theory," Nucl. Phys. (Proc. Suppl.) 46, 30 (1996).

${ }^{30}$ J. H. Schwarz, "The power of M theory," Phys. Lett. B 367, 97 (1996). 